\title{
Spontaneous Tumor Lysis Syndrome: A Rare Presentation in Breast Cancer
}

\author{
Muhammad Idrees $^{\mathrm{a}, \mathrm{b}}$, Saeeda Fatima ${ }^{\mathrm{a}}$, Eric Bravin ${ }^{\mathrm{a}}$
}

\begin{abstract}
Tumor lysis syndrome (TLS) is considered an oncologic emergency which requires early diagnosis and treatment to prevent mortality and morbidity. It refers to the constellation of metabolic disturbances manifested by acute renal failure associated with hyperuricemia, hyperkalemia, hypocalcemia and hyperphosphatemia. Diagnosis is made based on Cairo-Bishop clinical and laboratory criteria. Spontaneous TLS, occurring prior to the initiation of cancer therapy, has been reported in hematologic malignancies like high grade non-Hodgkin's lymphoma (NHL) and acute leukemia, and rarely in solid tumors including colon cancer, hepatocellular carcinoma and in at least two cases of breast cancer. We present a case of spontaneous TLS in breast cancer patient which is rare but interesting disease manifestation.
\end{abstract}

Keywords: Tumor; Breast; Adenocarcinoma; Tumor lysis; Syndrome; Spontaneous; Oncologic emergencies; TLS

\section{Introduction}

Tumor lysis syndrome (TLS) refers to rapid lysis of malignant cells following chemotherapy leading to metabolic disturbances which include hyperuricemia, hyperkalemia, hyperphosphatemia, and/or hypocalcemia and can lead to renal failure, cardiac arrhythmia, seizure or death [1]. It is usually seen in patients with hematologic malignancies.

Spontaneous TLS, prior to the initiation of cancer therapy, is not common and has only been reported in hematologic malignancies like high grade non-Hodgkin's lymphoma (NHL) and acute leukemia. It is very rare to find spontaneous TLS in patient with breast cancer especially without any chemotherapeutic intervention.

\section{Case Report}

A 48-year-old female with history significant for hypertension

Manuscript submitted December 20, 2018, accepted January 3, 2019

aBassett Medical Center, 1Atwel Road, Cooperstown, NY 13326, USA ${ }^{\mathrm{b}}$ Corresponding Author: Muhammad Idrees, Bassett Medical Center, 1Atwel Road, Cooperstown, NY 13326, USA. Email: idreessoomro@gmail.com

doi: https://doi.org/10.14740/jmc3237 and a recent abnormal mammogram for calcifications, presented with complaints of abdominal pain, nausea and jaundice. She reported abdominal pain for 2 weeks associated with yellowish discoloration of skin and nausea. She also reported decreased frequency of urination. No fever or chills was reported. On physical examination, her blood pressure was $90 / 57 \mathrm{~mm}$ $\mathrm{Hg}$ and was afebrile. Other significant examination findings include jaundice, $4 \times 4 \mathrm{~cm}$ right breast lump and abdominal tenderness in the epigastric area.

Laboratory examination revealed LD 1,437 U/L, potassium $6.2 \mathrm{mEq} / \mathrm{L}$, uric acid $>20 \mathrm{mg} / \mathrm{dL}$, anion gap $17 \mathrm{mEq} / \mathrm{L}$, blood urea nitrogen (BUN) $135 \mathrm{mg} / \mathrm{dL}$, creatinine $4.4 \mathrm{mg} / \mathrm{dL}$, calcium $9.8 \mathrm{mg} / \mathrm{dL}$, alkaline phosphatase $318 \mathrm{IU} / \mathrm{L}$, aspartate aminotransferase (AST) 1,211 U/L, alanine aminotransferase (ALT) $161 \mathrm{U} / \mathrm{L}$, total bilirubin $8.9 \mathrm{mg} / \mathrm{dL}$, albumin $2.4 \mathrm{~g} / \mathrm{dL}$, Mg $2.9 \mathrm{mg} / \mathrm{dL}$, phosphorus $5.1 \mathrm{mg} / \mathrm{dL}$ and international normalized ratio (INR) 1.6.

The diagnosis of TLS was made based on the clinical findings and chemistry data based on Cairo- Bishop Criteria (Table 1) and suspicion of malignancy based on imaging studies. She was treated with intravenous fluid, rasburicase and later with renal replacement therapy with improvement in clinical and laboratory findings.

Breast biopsy was done to evaluate the breast lump which revealed right breast infiltrating carcinoma $(\mathrm{ER}+, \mathrm{PR}+, \mathrm{Her}-2$ negative, P53 negative and Ki67 10\%). Further laboratory and radiographic studies were done for staging of the cancer and metastasis. She was noted to have metastatic lesions in liver and bone and was started on paclitaxel for initial treatment.

\section{Discussion}

TLS is medical emergency which occurs in certain type of tumors either spontaneously or after chemotherapy resulting

Table 1. Cairo-Bishop Definition of Laboratory Tumor Lysis Syndrome

\begin{tabular}{lll}
\hline Variable & Value & $\begin{array}{l}\text { Change from } \\
\text { baseline value }\end{array}$ \\
\hline Uric acid & $>8 \mathrm{mg} / \mathrm{dL}$ & $25 \%$ increase \\
Potassium & $>6 \mathrm{mEq} / \mathrm{L}$ & $25 \%$ increase \\
Phosphorus & $>4.5 \mathrm{mg} / \mathrm{dL}$ in adults & $25 \%$ increase \\
Calcium & $<7 \mathrm{mg} / \mathrm{dL}$ & $25 \%$ decrease \\
\hline
\end{tabular}

Adapted from Cairo et al [4]. 
Table 2. Cairo-Bishop Grading of Clinical Tumor Lysis Syndrome for Adults

\begin{tabular}{|c|c|c|c|c|c|c|}
\hline Variable & Grade 0 & Grade 1 & Grade 2 & Grade 3 & Grade 4 & Grade 5 \\
\hline Creatine & None & 1.5 times ULN & $>1.5-3.0$ times ULN & $>3.0-6.0$ times ULN & $>6.0$ times ULN & Death \\
\hline $\begin{array}{l}\text { Cardiac } \\
\text { arrhythmia }\end{array}$ & None & $\begin{array}{l}\text { Intervention } \\
\text { not indicated }\end{array}$ & $\begin{array}{l}\text { Non-urgent medical } \\
\text { intervention indicated }\end{array}$ & $\begin{array}{l}\text { Symptomatic and } \\
\text { incompletely controlled } \\
\text { medically or controlled } \\
\text { with device (e.g., } \\
\text { defibrillator) }\end{array}$ & $\begin{array}{l}\text { Life-threatening (e.g., } \\
\text { arrhythmia associated } \\
\text { with heart failure (HF), } \\
\text { hypotension, syncope, } \\
\text { shock). }\end{array}$ & Death \\
\hline
\end{tabular}

Adapted from Cairo et al [4]. ULN: upper limit of normal.

in release of intracellular content leading to severe metabolic abnormalities and organ dysfunction including acute kidney injury, cardiac arrhythmias and neuromuscular symptoms [2].

According to Cairo- Bishop Criteria (Table 1), laboratory TLS is defined as the presence of at least two or more biochemical variables within the 3 days before chemotherapy or 7 days after chemotherapy. Clinical TLS is defined as laboratory TLS plus one or more of the following that was not directly or probably attributable to a therapeutic agent: increased serum creatinine concentration $(=1.5$ times the upper limit of normal (ULN)), cardiac arrhythmia/sudden death, or a seizure [3].

Our patient met the diagnostic criteria of laboratory TLS (Table 1), as the three laboratory criteria were present and the worsening of acute renal failure to grade I meets to the definition of clinical TLS criteria established by Cairo and Bishop in 2004 [4] (Table 2)

Tumors most frequently associated with TLS are clinically aggressive non-Hodgkin lymphomas (NHLs) and acute lymphoblastic leukemia (ALL), particularly Burkitt lymphoma/ leukemia [5-7]. TLS has been rarely described after treatment of some non-hematologic solid tumors including breast cancer, lung cancer and colon cancer. The prognosis of TLS is worse in the case of solid tumors compared to hematology malignancy, which may be due to the frequent lack of early recognition and prevention $[1,8]$.

To the best of our knowledge, this is the second case report in the literature about spontaneous TLS occurring in a breast cancer patient without any treatment. However, our case is unique and quite different from the quoted paper, as our patient did not have reported diagnosis of breast cancer and presented with abnormal mammographic findings on initial presentations [9].

TLS management involves identifying high risk patients, initiation of preventive measures in risk patients and early identification of TLS and prompt initiation of supportive measures. Risk factors for TLS include bulky disease, extensive metastases, high proliferative rate, high sensitivity to anticancer therapy, type of anticancer therapy (e.g., using a combination therapy), elevated LDH, serum creatinine, uric acid, phos- phate at baseline, low urinary flow, dehydration, preexisting nephropathy, extrinsic compression of the urinary tract by the tumor, and exposure to nephrotoxins [10]. Some studies suggest that metastatic disease and particularly metastases in the liver should be a relevant risk factor, irrespective of the liver function [11].

Effective management involves the combination of treating specific electrolyte abnormalities, and/or acute renal failure, and the use of allopurinol, a xanthine oxidase inhibitor administered to reduce the conversion of nucleic acid byproducts to uric acid in order to prevent urate nephropathy and subsequent oliguric renal failure $[3,10]$. Rasburicase is better for the acute treatment of acute kidney injury (AKI) in TLS. Patients treated with rasburicase have shorter stays in the intensive care unit compared to allopurinol-treated patients [12].

Development of AKI associated with TLS is a strong predictor of death $[13,14]$. Irrespective of the cancer type, there is a $20-50 \%$ increase in mortality for undiagnosed or late-diagnosed TLS in solid tumors [15]. The best management for TLS is prevention.

Our patient received timely and aggressive hydration and was initiated on rasburicase and received renal replacement therapy for the acute kidney dysfunction.

\section{Conclusions}

We describe a case of spontaneous TLS as an initial presentation in breast cancer patient. We suggest considering TLS as diagnosis in patient with known or suspected breast malignancy with new onset kidney dysfunction and should be further investigated to prevent the morbidity and mortality associated with this severe disease.

\section{Consent}

Informed consent was obtained from the patient about the use of clinical information for the educational and publication pur- 
pose.

\section{References}

1. Mirrakhimov AE, Ali AM, Khan M, Barbaryan A. Tumor Lysis Syndrome in Solid Tumors: An up to Date Review of the Literature. Rare Tumors. 2014;6(2):5389.

2. Abu-Alfa AK, Younes A. Tumor lysis syndrome and acute kidney injury: evaluation, prevention, and management. Am J Kidney Dis. 2010;55(5 Suppl 3):S1-13; quiz S14-19.

3. Mirrakhimov AE, Voore P, Khan M, Ali AM. Tumor lysis syndrome: A clinical review. World J Crit Care Med. 2015;4(2):130-138.

4. Wilson FP, Berns JS. Tumor lysis syndrome: new challenges and recent advances. Adv Chronic Kidney Dis. 2014;21(1):18-26.

5. Gogia A, Raina V, Iqbal N, Murugan V. Spontaneous tumor lysis syndrome in a patient of chronic lymphocytic leukemia. Indian J Med Paediatr Oncol. 2014;35(1):120.

6. Jasek AM, Day HJ. Acute spontaneous tumor lysis syndrome. Am J Hematol. 1994;47(2):129-131.

7. Riccio B, Mato A, Olson EM, Berns JS, Luger S. Spontaneous tumor lysis syndrome in acute myeloid leukemia: two cases and a review of the literature. Cancer Biol Ther. 2006;5(12):1614-1617.

8. Mott FE, Esana A, Chakmakjian C, Herrington JD. Tu- mor lysis syndrome in solid tumors. Support Cancer Ther. 2005;2(3):188-191.

9. Spontaneous recurrent tumor lysis syndrome in breast cancer. American Journal of Clinical Oncology [Internet]. [cited 2018 Mar 8]. Available from: https://journals.lww. com/amjclinicaloncology/abstract/1995/02000/spontaneous_recurrent tumor_lysis_syndrome in.15.aspx.

10. Horl WH. [Tumor lysis syndrome: risk factors and treatment]. Wien Klin Wochenschr. 2005;117(1-2):7-17.

11. Zivin SP, Elias Y, Ray CE, Jr. Tumor lysis syndrome and primary hepatic malignancy: case presentation and review of the literature. Semin Intervent Radiol. 2015;32(1):3-9.

12. Eaddy M, Seal B, Tangirala M, Davies EH, O'Day K. Economic comparison of rasburicase and allopurinol for treatment of tumor lysis syndrome in pediatric patients. Am J Health Syst Pharm. 2010;67(24):2110-2114.

13. Serum uric acid and acute kidney injury: A mini review Science Direct [Internet]. [cited 2018 Apr 30]. Available from: https:/www.sciencedirect.com/science/article/pii/ S2090123216300741.

14. Darmon M, Guichard I, Vincent F, Schlemmer B, Azoulay E. Prognostic significance of acute renal injury in acute tumor lysis syndrome. Leuk Lymphoma. 2010;51(2):221227.

15. Predictorsofin-HospitalMortalityinTumorLysis Syndrome | Blood Journal [Internet]. [cited 2018 Apr 30]. Available from: http://www.bloodjournal.org/content/124/21/4862? sso-checked=true. 